\title{
ZSM-5 催化绿色合成烯胺酮化合物
}

\section{魏振中肖兰王永秋李品华*}

(淮北师范大学化学与材料科学学院 淮北 235000)

\begin{abstract}
摘要 以环境友好的 ZSM-5 分子篮为催化剂, 催化 $\beta$-二羰基化合物与胺反应, 绿色合成 $\beta$-烯胺酮类化合物. 该方法环 境友好、处理方法简单、收率高、催化剂可回收使用.

关键词 催化; 烯胺酮; ZSM-5 分子篮
\end{abstract}

\section{Green Synthesis of Enaminones Catalyzed by ZSM-5}

\author{
Wei, Zhenzhong Xiao, Lan Wang, Yongqiu Li, Pinhua* \\ (School of Chemistry and Materials Science, Huaibei Normal University, Huaibei 235000)
}

\begin{abstract}
It was found that ZSM-5 as a green catalyst could efficiently catalyze the reaction of various primary amines with $\alpha, \beta$-dicarboxyl compounds to afford $\beta$-enaminones in good yields at room temperature. The method provides a novel, simple and convenient route to substitute $\beta$-enaminones from primary amines and $\alpha, \beta$-dicarboxyl compounds. The catalyst could also be recovered easily.
\end{abstract}

Keywords catalysis; $\beta$-enaminones; ZSM- 5 zeolite

$\beta$-烯胺酮是合成肽 ${ }^{[1]}$ 、生物碱 ${ }^{[2 \sim 5]}$ 和吡唑 ${ }^{[6]}$ 等生物活 性和药理活性物质的重要中间体, 该类化合物的合成一 直是人们研究的焦点 ${ }^{[7,8]} . \beta$-烯胺酮的传统合成方法是在 盐酸等质子酸催化下 1,3 -二羰基化合物和胺反应, 将反 应中生成的水蒸出提高产率，但该法存在反应温度高、 产率低、污染环境和后处理麻烦等缺点. 因此, 人们对 $\beta$-烯胺酮的合成方法和高效催化剂进行了广泛深入的 研究, 已报道的部分催化剂有: $\mathrm{CuCl}^{[9]}, \mathrm{CeCl}_{3} \cdot 7 \mathrm{H}_{2} \mathrm{O}^{[10]}$, $\mathrm{NaAuCl}_{4}{ }^{[11]}, \mathrm{Zn}\left(\mathrm{ClO}_{4}\right)_{2} \cdot 6 \mathrm{H}_{2} \mathrm{O}^{[12]} \mathrm{AcOH}^{[13]}, \mathrm{InBr}_{3}{ }^{[14]}, \mathrm{BF}_{3} \bullet$ $\mathrm{OEt}_{2}{ }^{[15]}, \quad \mathrm{Bi}(\mathrm{TFA})_{3}{ }^{[16]} \quad \mathrm{Al}_{2} \mathrm{O}_{3}{ }^{[17]}, \quad \mathrm{NaHSO}_{4} \cdot \mathrm{SiO}_{2}{ }^{[18]}$ $\mathrm{Yb}(\mathrm{OTf})_{3}{ }^{[19]}, \mathrm{H}_{3} \mathrm{PW}_{12} \mathrm{O}_{40}(\mathrm{PW})^{[20]}, \quad \mathrm{VO}(\mathrm{acac})_{2}^{[21]}$, silica chloride ${ }^{[22]}, \mathrm{HClO}_{4} \cdot \mathrm{SiO}_{2}{ }^{[23]}, \mathrm{Fe}\left(\mathrm{HSO}_{4}\right)_{3} \cdot \mathrm{SiO}_{2}{ }^{[24]}$, Cu-nanop$\operatorname{articles}^{[25]}$, Silver nanoparticles ${ }^{[26]}$. 合成方法上报道有微 波 ${ }^{[27]}$ 和超声波 ${ }^{[28,29]}$ 高频振荡技术 ${ }^{[30]}$ 等作为辅助手段, 这些研究都取得了较满意的结果. 但改进的催化体系均 存在一些缺点, 如反应时间长, 产率低, 反应条件苛刻, 催化剂价格较昂贵, 使用挥发溶剂且不具经济性. 因此, 开发高效、廉价的催化剂, 应用于催化胺与 1,3-二羰基 化合物的缩合反应, 具有重要的理论和现实意义. 本文 首次将 ZSM-5 分子篮应用催化该反应得到了比较理想 的结果.
ZSM-5 作为一种无毒、廉价催化剂被广泛应用于有 机合成反应 ${ }^{[31,32]}, Z S M-5$ 分子篮被认为是目前最具工业 应用前景的催化剂, 是一种具有独特的三维孔道结构、 高比表面积和良好的择形性的固体酸催化剂, 同时 ZSM-5 分子篮具有良好的耐酸性、热稳定性、水蒸气稳 定性. 并且 ZSM-5 分子篎价格便宜、易于回收、绿色环 保，在使用过程中安全、稳定、操作简便. 合成路线如 Eq. 1 所示.

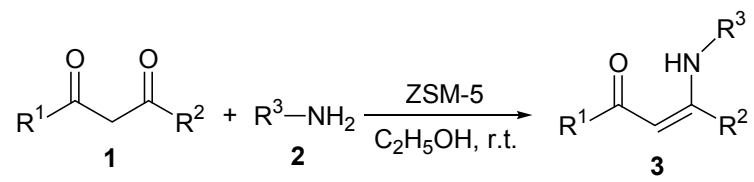

\section{1 结果与讨论}

$\beta$-烯胺酮及其衍生物的合成方法有很多种，但是大 部分局限性比较大, 我们选用价格便宜, 易回收的 ZSM-5 分子篮作为催化剂, 取得了非常好的效果, 以对 甲苯胺和乙酰丙酮反应为例探究了 ZSM-5 分子篮硅铝 比和乙酰丙酮用量对反应的影响, ZSM-5 分子篮硅铝比 越大酸性越强, 不同硅铝比的 ZSM-5 催化合成 $\beta$-烯胺 酮的结果如表 1 .

*E-mail: pphuali@126.com

Received January 8, 2014; revised March 10, 2014; published online March 26, 2014. 
表 1 催化剂硅铝比对收率的影响 ${ }^{a}$

Table 1 Effect of $\mathrm{Si} / \mathrm{Al}$ ratio on yield

\begin{tabular}{|c|c|c|c|}
\hline 序号 & $n(\mathrm{Si}) / n(\mathrm{Al})$ & $n$ (胺) $/ n$ (乙酰丙酮) & 产率/\% \\
\hline 1 & 25 & $1: 1$ & 80 \\
\hline 2 & 38 & $1: 1$ & 87 \\
\hline 3 & 50 & $1: 1$ & 94 \\
\hline 4 & 80 & $1: 1$ & 95 \\
\hline 5 & 80 & $1.05: 1$ & 95 \\
\hline 6 & 80 & $1: 1.05$ & 96 \\
\hline 7 & 80 & $1: 1.1$ & 97 \\
\hline 8 & 80 & $1: 1.15$ & 97 \\
\hline 9 & 100 & $1: 1$ & 95 \\
\hline
\end{tabular}

由表 1 可以发现随着硅铝比的增加反应收率逐渐升 高, 但是达到硅铝比 50 以后对产率的影响不大, 由此可 见酸性大小对反应的影响较大, 但是当酸性增加到一定 程度后即不在是反应的主要影响因素.

在此基础上选用硅铝比 80 的 ZSM-5 分子笁继续以 对甲苯胺和苯甲酰丙酮考察了 ZSM-5 分子笁用量对催 化反应的影响. 结果如表 2 .

表 2 催化剂用量对收率的影响 ${ }^{a}$

Table 2 Effect of catalyst dosage on yield

\begin{tabular}{ccc}
\hline 序号 & 催化剂用量/equiv. & 产率 $/ \%$ \\
\hline 1 & 0.05 & 92 \\
2 & 0.10 & 94 \\
3 & 0.15 & 98 \\
4 & 0.20 & 97 \\
5 & 0.25 & 96 \\
6 & 0.30 & 91 \\
\hline
\end{tabular}

${ }^{a} 2 \mathrm{mmol}$ 对甲苯胺, $2.2 \mathrm{mmol}$ 乙酰丙酮, $0.15 \mathrm{~g}$ 硅铝比 $80 \mathrm{ZSM}-5,10 \mathrm{~mL}$ 无 水乙醇, 室温摚拌 $10 \mathrm{~h}$.

由表 2 可以看出催化剂的量增加到 $0.15 \mathrm{~g}$ 以上后可 能由于催化剂量的增加导致对产品的吸附使得产率的 下降, 我们选用催化剂的用量为 $0.15 \mathrm{~g}$. 在优化的反应 条件下, 对 $0.15 \mathrm{~g}$ 硅铝比 80 的 ZSM-5 催化剂进行了回 收套用实验见表 3 , 套用 5 次未见催化剂活性明显下降, 并对回收的催化剂与催化剂原样进行对比, 见图 2. 通 过对比发现, 由于在催化反应过程中的摚拌作用使得催 化剂颗粒更加细小, 其粉末衍射强度有所减弱, 但是两 者的粉末衍射位置几乎是完全一致的, 这说明催化剂在 反应前后的结构并没有变化.

为进一步验证反应的可行性, 我们对底物进行拓 展，并对部分化合物进行放大 10 倍实验(表 4). 其中 3d, 3e, 3g, 3j, 3k, 30 为未见报道化合物. 通过 ${ }^{1} \mathrm{H}$ NMR 可以 清楚地分辨出乙酰丙酮和苯甲酰丙酮与胺生成的产物 为顺式, $\mathrm{N}-\mathrm{H}$ 吸收峰受分子内氢键影响出现在低场, 而环酮 1,3-环己二酮和 5,5-二甲基-1,3-环已二酮与胺反 应生成产物 $\mathrm{N}-\mathrm{H}$ 吸收峰出现在高场, 可能是由于六元
环的张力分子内不能形成氢键(图 3).

表 3 催化剂回收套用情况 ${ }^{a}$

Table 3 Results of catalyst recycle applied

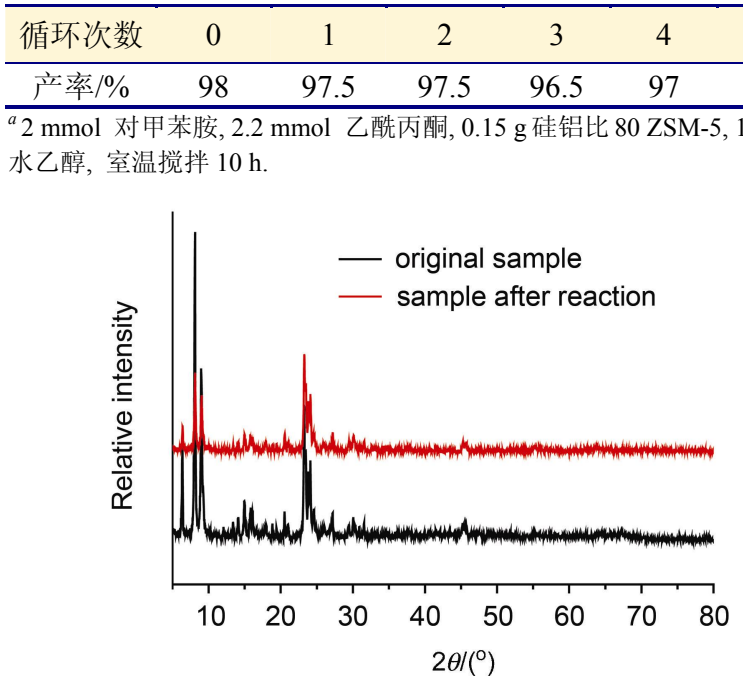

图 $2 \mathrm{ZSM}-5$ 的 PXRD 图

Figure 2 PXRD patterns of ZSM-5

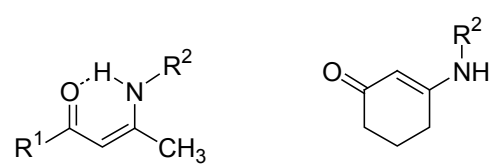

Scheme 1

\section{2 结论}

本文首次报道 ZSM-5 分子笁催化伯胺与 1,3-二羰 基化合物缩合生成 $\beta$-烯胺酤. 该方法反应试剂易得、操 作方法简单、后处理方便、反应条件温和、操作简单、 催化剂廉价易得且能回收套用. 为 $\beta$-烯胺酮类化合物的 合成寻找到了一种简捷绿色的方法.

\section{3 实验部分}

\section{1 仪器与试剂}

WPS-1 型数字熔点仪(温度计未校正); Bruker 核磁 共振仪(TMS 为内标, $400 \mathrm{~Hz}$ ); BRUKER D8 ADVANCE 型 $X$ 射线衍射仪(扫描电压电流分别为 $40 \mathrm{kV}, 40 \mathrm{~mA}$, $\lambda=0.15418 \mathrm{~nm}$, 扫描范围 $2 \theta=5^{\circ} \sim 80^{\circ}$ ). PE-2400 型元 素自动分析仪; 所用试剂均为 AR 级, ZSM-5 分子篮外 购与南开大学催化剂厂 (120 目).

\section{2 实验方法}

\subsection{1 $\beta$-烯胺酮 3 合成步骤}

在 $25 \mathrm{~mL}$ 的反应瓶中加入对甲苯胺 $(2 \mathrm{mmol}, 214$ $\mathrm{mg}$ )、乙酰丙酮 $(2.2 \mathrm{mmol}, 220 \mathrm{mg}) 、 0.15 \mathrm{~g}$ 硅铝比 80 的 ZSM-5 分子笁, 无水乙醇 $10 \mathrm{~mL}$, 室温下搅拌, TLC 监测 反应进程, $10 \mathrm{~h}$ 后原料反应完全, 停止反应, 加热反应 
表 4 ZSM-5 催化合成 $\beta$-烯胺酮 ${ }^{a}$

Table 4 Catalytic synthesis of $\beta$-enaminones with ZSM-5

\begin{tabular}{|c|c|c|c|c|c|c|c|}
\hline \multirow{2}{*}{ Compd. } & \multirow{2}{*}{$\mathrm{R}^{1}$} & \multirow{2}{*}{$\mathrm{R}^{2}$} & \multirow{2}{*}{$\mathrm{R}^{3}$} & \multirow{2}{*}{ Time/h } & \multirow{2}{*}{ Yield/\% } & \multicolumn{2}{|c|}{ m.p. $/{ }^{\circ} \mathrm{C}$} \\
\hline & & & & & & Actual & Reference \\
\hline $3 a$ & $\mathrm{CH}_{3}$ & $\mathrm{CH}_{3}$ & $p-\mathrm{CH}_{3} \mathrm{C}_{6} \mathrm{H}_{4}$ & 10 & 98 & $66 \sim 67$ & $65 \sim 67^{[9]}$ \\
\hline $3 \mathbf{b}$ & $\mathrm{CH}_{3}$ & $\mathrm{CH}_{3}$ & $p-\mathrm{ClC}_{6} \mathrm{H}_{4}$ & 12 & 92 & $60 \sim 61$ & $58 \sim 60^{[9]}$ \\
\hline $3 c$ & $\mathrm{CH}_{3}$ & $\mathrm{CH}_{3}$ & $\mathrm{C}_{6} \mathrm{H}_{5}$ & 12 & 90 & $48 \sim 49$ & $48 \sim 49^{[9]}$ \\
\hline 3d & $\mathrm{CH}_{3}$ & $\mathrm{CH}_{3}$ & $o-\mathrm{HOC}_{6} \mathrm{H}_{4}$ & 7 & 96 & $193 \sim 194$ & \\
\hline $3 e$ & $\mathrm{CH}_{3}$ & $\mathrm{CH}_{3}$ & $\mathrm{CH}_{3} \mathrm{CH}_{2} \mathrm{CH}_{2}$ & 6 & 94 & & \\
\hline $3 f$ & $\mathrm{C}_{6} \mathrm{H}_{5}$ & $\mathrm{CH}_{3}$ & $p-\mathrm{CH}_{3} \mathrm{C}_{6} \mathrm{H}_{4}$ & 8 & 97 & $90 \sim 91$ & $90 \sim 92^{[24]}$ \\
\hline $3 g$ & $\mathrm{C}_{6} \mathrm{H}_{5}$ & $\mathrm{CH}_{3}$ & $m-\mathrm{HOC}_{6} \mathrm{H}_{4}$ & 5 & 98 & $156 \sim 157$ & \\
\hline $3 h$ & \multicolumn{2}{|c|}{$\mathrm{CH}_{2} \mathrm{CH}_{2} \mathrm{CH}_{2}$} & $\mathrm{C}_{6} \mathrm{H}_{5}$ & 12 & 91 & $178 \sim 180$ & $179 \sim 180^{[19]}$ \\
\hline $3 \mathbf{i}$ & \multicolumn{2}{|c|}{$\mathrm{CH}_{2} \mathrm{CH}_{2} \mathrm{CH}_{2}$} & $p-\mathrm{ClC}_{6} \mathrm{H}_{4}$ & 13 & 92 & $191 \sim 192$ & $191 \sim 192^{[24]}$ \\
\hline $3 \mathbf{j}$ & \multicolumn{2}{|c|}{$\mathrm{CH}_{2} \mathrm{CH}_{2} \mathrm{CH}_{2}$} & $o-\mathrm{CH}_{3} \mathrm{OC}_{6} \mathrm{H}_{4}$ & 9 & 96 & $127 \sim 128$ & \\
\hline $3 \mathbf{k}$ & \multicolumn{2}{|c|}{$\mathrm{CH}_{2} \mathrm{CH}_{2} \mathrm{CH}_{2}$} & $m-\mathrm{HOC}_{6} \mathrm{H}_{4}$ & 8 & 98 & $180 \sim 181$ & \\
\hline 31 & \multicolumn{2}{|c|}{$\mathrm{CH}_{2} \mathrm{C}\left(\mathrm{CH}_{3}\right)_{2} \mathrm{CH}_{2}$} & $p-\mathrm{CH}_{3} \mathrm{C}_{6} \mathrm{H}_{4}$ & 11 & 97 & $198 \sim 199$ & $199 \sim 201^{[24]}$ \\
\hline $3 m$ & \multicolumn{2}{|c|}{$\mathrm{CH}_{2} \mathrm{C}\left(\mathrm{CH}_{3}\right)_{2} \mathrm{CH}_{2}$} & $p-\mathrm{ClC}_{6} \mathrm{H}_{4}$ & 14 & 94 & $208 \sim 209$ & $208 \sim 209^{[24]}$ \\
\hline $3 n$ & \multicolumn{2}{|c|}{$\mathrm{CH}_{2} \mathrm{C}\left(\mathrm{CH}_{3}\right)_{2} \mathrm{CH}_{2}$} & $\mathrm{C}_{6} \mathrm{H}_{5}$ & 13 & 92 & $184 \sim 185$ & $184 \sim 185^{[24]}$ \\
\hline 30 & \multicolumn{2}{|c|}{$\mathrm{CH}_{2} \mathrm{C}\left(\mathrm{CH}_{3}\right)_{2} \mathrm{CH}_{2}$} & $m-\mathrm{HOC}_{6} \mathrm{H}_{4}$ & 7 & 97 & $240 \sim 241$ & \\
\hline
\end{tabular}

a 放大实验: 3a, Time: 11.5 h, Yield: 98.5\%; 3f, Time: 8.5 h, Yield: 97\%; 3j, Time: 9 h, Yield: 97.5\%; 3l, Time: 11.5 h, Yield: $97 \%$.

液后趁热抽滤回收催化剂, 少量热乙醇洗涤催化剂, 合 并滤液，将滤液减压浓缩后经柱层析分离 $[V$ (石油醚)： $V($ 乙酸乙酯 $)=5: 1]$, 得烯胺酮化合物 $\mathbf{3 a}$.

4-对甲苯胺基-3-戊烯-2-酮(3a): ${ }^{1} \mathrm{H}$ NMR (400 MHz, $\left.\mathrm{CDCl}_{3}\right) \delta: 12.40(\mathrm{~s}, 1 \mathrm{H}), 7.05 \sim 7.23(\mathrm{~m}, 4 \mathrm{H}), 5.17(\mathrm{~s}, 1 \mathrm{H})$, $2.34(\mathrm{~s}, 3 \mathrm{H}), 2.08(\mathrm{~s}, 3 \mathrm{H}), 1.96(\mathrm{~s}, 3 \mathrm{H}) ;{ }^{13} \mathrm{C}$ NMR $(100$ $\left.\mathrm{MHz}, \mathrm{CDCl}_{3}\right) \delta: 195.75,160.50,135.99,129.53,124.74$, 97.08, 28.96, 20.75, 19.59 .

4-对氯苯胺基-3-戊烯-2-酮(3b): ${ }^{1} \mathrm{H}$ NMR $(400 \mathrm{MHz}$, $\left.\mathrm{CDCl}_{3}\right) \delta: 12.42(\mathrm{~s}, 1 \mathrm{H}), 7.32 \sim 7.16(\mathrm{~m}, 4 \mathrm{H}), 5.20(\mathrm{~s}, 1 \mathrm{H})$, 2.09 (s, 3H), 1.97 (s, 3H); $\left.{ }^{13} \mathrm{C} \mathrm{NMR} \mathrm{(100} \mathrm{MHz,} \mathrm{CDCl}_{3}\right) \delta$ : $189.11,159.55,137.27,130.85,129.09,125.73,98.00$, $79.89,29.11,19.65$.

4-苯胺基-3-戊烯-2-酮(3c): ${ }^{1} \mathrm{H}$ NMR (400 MHz, $\left.\mathrm{CDCl}_{3}\right) \delta: 12.49(\mathrm{~s}, 1 \mathrm{H}), 7.45 \sim 7.03(\mathrm{~m}, 5 \mathrm{H}), 5.20(\mathrm{~s}, 1 \mathrm{H})$, 2.08 (s, 3H), $2.00(\mathrm{~s}, 3 \mathrm{H}) ;{ }^{13} \mathrm{C}$ NMR $\left(100 \mathrm{MHz}, \mathrm{CDCl}_{3}\right) \delta$ : $196.05,160.13,138.63,128.98,125.45,124.66,97.49$, $29.08,19.74$

4-间羟基苯胺基-3-戊烯-2-酮(3d): 淡黄色固体; ${ }^{1} \mathrm{H}$ NMR (400 MHz, DMSO- $\left.d_{6}\right) \delta: 12.12(\mathrm{~s}, 1 \mathrm{H}), 9.86(\mathrm{~s}, 1 \mathrm{H})$, $7.23 \sim 6.66(\mathrm{~m}, 4 \mathrm{H}), 5.13(\mathrm{~s}, 1 \mathrm{H}), 1.95(\mathrm{~s}, 6 \mathrm{H}) ;{ }^{13} \mathrm{C} \mathrm{NMR}$ $\left(100 \mathrm{MHz}\right.$, DMSO- $\left.d_{6}\right) \delta: 194.57,160.36,150.75,126.50$, $119.41,97.51,40.13,39.84,29.20,19.80$. Anal. calcd for $\mathrm{C}_{11} \mathrm{H}_{13} \mathrm{NO}_{2}$ : C 69.09, H 6.85, N 7.32; found C 69.02, $\mathrm{H}$ $6.80, \mathrm{~N} 7.35$

4-正丙胺基-3-戊烯-2-酮(3e)：黄色液体; ${ }^{1} \mathrm{H}$ NMR $\left(400 \mathrm{MHz}, \mathrm{CDCl}_{3}\right) \delta: 10.78(\mathrm{~s}, 1 \mathrm{H}), 5.07 \sim 4.70(\mathrm{~m}, 1 \mathrm{H})$, $3.25 \sim 2.93(\mathrm{~m}, 2 \mathrm{H}), 2.01 \sim 1.80(\mathrm{~m}, 6 \mathrm{H}), 1.40 \sim 1.60(\mathrm{~m}$, 2H), $1.01 \sim 0.77(\mathrm{~m}, 3 \mathrm{H}) ;{ }^{13} \mathrm{C} \mathrm{NMR}\left(101 \mathrm{MHz}, \mathrm{CDCl}_{3}\right) \delta$ :
194.30, 163.02, 94.80, 23.17, 11.15. Anal. calcd for $\mathrm{C}_{8} \mathrm{H}_{15^{-}}$ NO: C 68.04, H 10.71, N 9.92; found C 68.02, H 10.70, N 9.96.

4-对甲苯胺基-4-苯基-3-丁烯-2-酮(3f): ${ }^{1} \mathrm{H}$ NMR $\left(400 \mathrm{MHz}, \mathrm{CDCl}_{3}\right) \delta: 13.04(\mathrm{~s}, 1 \mathrm{H}), 7.87 \sim 7.41(\mathrm{~m}, 5 \mathrm{H})$, $7.21 \sim 7.05(\mathrm{~m}, 4 \mathrm{H}), 5.89(\mathrm{~s}, 1 \mathrm{H}), 2.37(\mathrm{~s}, 3 \mathrm{H}), 2.13(\mathrm{~s}$, $3 \mathrm{H}) ;{ }^{13} \mathrm{C}$ NMR $\left(100 \mathrm{MHz}, \mathrm{CDCl}_{3}\right) \delta: 188.38,162.45$, $140.03,129.62,93.78,20.81,20.22$.

4-间着基苯胺基-4-苯基-3-丁烯-2-酮(3g)：淡黄色固 体; ${ }^{1} \mathrm{H}$ NMR (400 MHz, $\mathrm{CDCl}_{3}$ ) $\delta: 13.00(\mathrm{~s}, 1 \mathrm{H}), 8.76$ (s, $1 \mathrm{H}), 7.90 \sim 7.32(\mathrm{~m}, 5 \mathrm{H}), 7.32 \sim 6.60(\mathrm{~m}, 4 \mathrm{H}), 5.89(\mathrm{~s}$, 1H), 2.16 (s, 3H); ${ }^{13} \mathrm{C}$ NMR (100 MHz, $\left.\mathrm{CDCl}_{3}\right) \delta: 188.95$, 131.03, 116.54, 111.78, 94.36, 20.31. Anal. calcd for $\mathrm{C}_{16} \mathrm{H}_{15} \mathrm{NO}_{2}$ : C 75.87, H 5.97, N 5.53; found C 75.82, H $5.98, \mathrm{~N} 5.52$.

3-苯胺基环已-2-烯酮 (3h): ${ }^{1} \mathrm{H}$ NMR $(400 \mathrm{MHz}$, $\left.\mathrm{CDCl}_{3}\right) \delta: 7.38 \sim 7.15(\mathrm{~m}, 5 \mathrm{H}), 6.93(\mathrm{~s}, 1 \mathrm{H}), 5.56(\mathrm{~s}, 1 \mathrm{H})$, $2.51(\mathrm{t}, J=6.1 \mathrm{~Hz}, 2 \mathrm{H}), 2.34(\mathrm{t}, J=6.4 \mathrm{~Hz}, 2 \mathrm{H}), 2.06 \sim$ $1.96(\mathrm{~m}, 2 \mathrm{H}) ;{ }^{13} \mathrm{C}$ NMR $\left(100 \mathrm{MHz}, \mathrm{CDCl}_{3}\right) \delta: 198.22$, $162.34,138.05,129.19,125.39,99.55,36.39,29.59,21.74$.

3-对氯苯胺基环已-2-烯酮(3i): ${ }^{1} \mathrm{H}$ NMR $(400 \mathrm{MHz}$, $\left.\mathrm{CDCl}_{3}\right) \delta: 7.36 \sim 7.07(\mathrm{~m}, 4 \mathrm{H}), 5.48(\mathrm{~s}, 1 \mathrm{H}), 2.50(\mathrm{t}, J=6.0$ $\mathrm{Hz}, 2 \mathrm{H}), 2.33(\mathrm{t}, J=6.4 \mathrm{~Hz}, 2 \mathrm{H}), 1.95 \sim 2.05(\mathrm{~m}, 2 \mathrm{H}) ;{ }^{13} \mathrm{C}$ NMR $\left(100 \mathrm{MHz}, \mathrm{CDCl}_{3}\right) \delta: 198.28,162.24,136.68$, $129.29,99.72,36.38,29.48,21.68$

3-邻甲氧基苯胺基环己-2-烯酮 (3j): 黄色固体; ${ }^{1} \mathrm{H}$ NMR $\left(400 \mathrm{MHz}, \mathrm{CDCl}_{3}\right) \delta: 7.38 \sim 6.80(\mathrm{~m}, 4 \mathrm{H}), 6.39(\mathrm{~s}$, $1 \mathrm{H}), 5.70(\mathrm{~s}, 1 \mathrm{H}), 3.83(\mathrm{~s}, 3 \mathrm{H}), 2.52(\mathrm{t}, J=6.0 \mathrm{~Hz}, 2 \mathrm{H})$, $2.35(\mathrm{t}, J=6.3 \mathrm{~Hz}, 2 \mathrm{H}), 1.96 \sim 2.06(\mathrm{~m}, 2 \mathrm{H}) ;{ }^{13} \mathrm{C}$ NMR 
$\left(100 \mathrm{MHz}, \mathrm{CDCl}_{3}\right) \delta: 198.25,160.92,127.32,110.91$, $100.25,55.51,36.39,30.01,21.74$. Anal. calcd for $\mathrm{C}_{13} \mathrm{H}_{15} \mathrm{NO}_{2}$ : C 71.87, $\mathrm{H}$ 6.96, N 6.45; found $\mathrm{C} 71.84, \mathrm{H}$ 6.98, N 6.42.

3-间羟基苯胺基环己-2-烯酮(3k): 黄色晶体; ${ }^{1} \mathrm{H}$ NMR (400 MHz, Acetone- $\left.d_{6}\right) \delta: 7.15(\mathrm{t}, J=8.0 \mathrm{~Hz}, 1 \mathrm{H})$, $6.76(\mathrm{~s}, 1 \mathrm{H}), 6.64(\mathrm{~m}, 2 \mathrm{H}), 5.60(\mathrm{~s}, 1 \mathrm{H}), 2.57(\mathrm{t}, J=6.1 \mathrm{~Hz}$, $2 \mathrm{H}), 2.25(\mathrm{t}, J=6.4 \mathrm{~Hz}, 2 \mathrm{H}), 2.03 \sim 1.89(\mathrm{~m}, 2 \mathrm{H}) ;{ }^{13} \mathrm{C}$ NMR (100 MHz, Acetone- $\left.d_{6}\right) \delta: 202.30,167.37,163.31$, 145.32, 134.97, 118.91, 103.88, 41.36, 26.86, 4.25. Anal. calcd for $\mathrm{C}_{12} \mathrm{H}_{13} \mathrm{NO}_{2}$ : C 70.92, H 6.45, N 6.89; found $\mathrm{C}$ 70.94, H 6.48, N 6.84.

3-对甲苯胺基-5,5-二甲基环已-2-烯酮(31)： ${ }^{1} \mathrm{H}$ NMR $\left(400 \mathrm{MHz}, \mathrm{CDCl}_{3}\right) \delta: 7.19 \sim 7.08(\mathrm{~m}, 4 \mathrm{H}), 6.50(\mathrm{~s}, 1 \mathrm{H})$, $5.51(\mathrm{~s}, 1 \mathrm{H}), 2.33(\mathrm{~s}, 5 \mathrm{H}), 2.19(\mathrm{~s}, 2 \mathrm{H}), 1.09(\mathrm{~s}, 6 \mathrm{H}) ;{ }^{13} \mathrm{C}$ NMR $\left(100 \mathrm{MHz}, \mathrm{CDCl}_{3}\right) \quad \delta: 197.60,160.70,135.44$, $129.73,98.21,50.25,43.39,32.70,28.20,20.79$.

3-对氯苯胺基-5,5-二甲基环已-2-烯酮 (3m): ${ }^{1} \mathrm{H}$ NMR (400 MHz, $\left.\mathrm{CDCl}_{3}\right) \delta: 7.37 \sim 7.04(\mathrm{~m}, 4 \mathrm{H}), 6.44(\mathrm{~s}$, $1 \mathrm{H}), 5.52(\mathrm{~s}, 1 \mathrm{H}), 2.34(\mathrm{~s}, 2 \mathrm{H}), 2.22(\mathrm{~s}, 2 \mathrm{H}), 1.11(\mathrm{~s}, 6 \mathrm{H})$; ${ }^{13} \mathrm{C}$ NMR $\left(100 \mathrm{MHz}, \mathrm{CDCl}_{3}\right) \delta: 198.01,136.69,129.35$, 124.94, 98.96, 50.24, 43.43, 32.72, 28.19.

3-苯胺基-5,5-二甲基环已-2-烯酮(3n): ${ }^{1} \mathrm{H}$ NMR $\left(400 \mathrm{MHz}, \mathrm{CDCl}_{3}\right) \delta: 7.38 \sim 7.04(\mathrm{~m}, 5 \mathrm{H}), 6.90(\mathrm{~s}, 1 \mathrm{H})$, $5.57(\mathrm{~s}, 1 \mathrm{H}), 2.35(\mathrm{~s}, 2 \mathrm{H}), 2.20(\mathrm{~s}, 2 \mathrm{H}), 1.08(\mathrm{~s}, 6 \mathrm{H}) ;{ }^{13} \mathrm{C}$ NMR (100 MHz, $\left.\mathrm{CDCl}_{3}\right) \delta: 197.87,138.17,129.18$, $125.34,98.29,50.25,43.37,32.70,28.19$.

3-间着基苯胺基-5,5-二甲基环已-2-烯酩(30): 黄色 晶体; ${ }^{1} \mathrm{H}$ NMR (400 MHz, DMSO- $\left.d_{6}\right) \delta: 9.54(\mathrm{~s}, 1 \mathrm{H}), 8.69$ $(\mathrm{s}, 1 \mathrm{H}), 7.27 \sim 6.77(\mathrm{~m}, 4 \mathrm{H}), 5.37(\mathrm{~s}, 1 \mathrm{H}), 2.35(\mathrm{~s}, 2 \mathrm{H})$, $2.04(\mathrm{~s}, 2 \mathrm{H}), 1.00(\mathrm{~s}, 6 \mathrm{H}) ;{ }^{13} \mathrm{C}$ NMR $\left(100 \mathrm{MHz}, \mathrm{DMSO}-d_{6}\right)$ $\delta: 195.81,160.22,158.30,140.62,130.20,113.72,97.41$, 42.47, 32.58, 28.29. Anal. calcd for $\mathrm{C}_{14} \mathrm{H}_{17} \mathrm{NO}_{2}$ : C 72.70, H 7.41, N 6.06; found C 72.68, H 7.44, N 6.04.

\section{References}

[1] Beholz, L. G.; Benovsky, P.; Ward, D. L.; Barta, N. S.; Stille, J. R. J. Org. Chem. 1997, 62, 1033.

[2] Michael, J. P.; Koning, C. B.; Hosken, G. D.; Stanbury, T. V. Tetrahedron 2001, 57, 9635.

[3] Bartoli, G.; Cimarelli, C.; Marcantoni, E.; Palmeiri, G.; Petrini, M. J. Org. Chem. 1994, 59, 5328.

[4] Barta, N. S.; Brode, A.; Stille, J. R. J. Am. Chem. Soc. 1994, 116, 6201.

[5] Michael, J. P.; Parsons, A. S. Tetrahedron 1999, 55, 10915.

[6] Valduga, C. J.; Braibante, H. S.; Braibante. E. F. J. Heterocycl. Chem. 1998, 35, 189.
[7] Li, M.; Guo, W.-S.; Wen, L.-R. Chin. J. Org. Chem. 2006, 26, 1192 (in Chinese). (李明, 郭维斯, 文丽荣, 有机化学, 2006, 26, 1192.)

[8] Li, A.-J.; Feng, B.; Liu, Q.-C. Chin. J. Org. Chem. 2011, 31, 106 (in Chinese). (李爱军, 冯宝, 刘倩春, 有机化学, 2011, 31, 106.)

[9] Qu, Y.-P.; Wang, L.-X.; Zhou, X.-Y. Chin. J. Org. Chem. 2013, 33, 860 (in Chinese). (矍益萍, 王龙翔, 周晓玉, 有机化学, 2013, 33, 860.)

[10] Khodaei, M. M.; Khosropour, A. R.; Kookhazadeh, M. Synlett 2004, 1980.

[11] Arcadi, A.; Bianchi, G.; Di Giuseppe, S.; Marinelli, F. Green Chem. 2003, 5,64

[12] Bartoli, G.; Bosco, M.; Locatelli, M.; Marcantoni, E.; Melchiorre, P.; Sambri, L. Synlett 2004, 239.

[13] Brandt, C. A.; Da Silva, A. C. M. P.; Pancote, C. G.; Brito, C. L.; Da Silveira, M. A. B. Synthesis 2004, 1557

[14] Zhang, Z. H.; Yin, L.; Wang, Y. M. Adv. Synth. Catal. 2006, 348, 184.

[15] Stefane, B.; Polanc, S. Synlett 2004, 698.

[16] Khosropour, A. R.; Khodaei, M. M.; Kookhazadeh, M. Tetrahedron Lett. 2004, 45, 1725.

[17] Valduga, C. J.; Braibante, H. S.; Braibante, E. F. J. J. Heterocycl. Chem. 1998, 35, 189

[18] Wng, H.-S.; Miao, J.-Y. Chin. J. Org. Chem. 2007, 27, 266 (in Chinese). (王宏社, 苗建英, 有机化学, 2007, 27, 266.)

[19] Francesco, E.; Salvatore, G.; Massimo, C. Tetrahedron Lett. 2007, $48,2717$.

[20] Ezzat, R.; Houri, M.; Sara, E.; Mohammad, J. Appl. Catal. A: Gen. 2009, 352, 202.

[21] Rajibul, A.; Naznin, A.; Mohammad, H. Tetrahedron Lett. 2013, 54, 436.

[22] Gholap, R.; Narayan. S.; Thomas. D. J. Mol. Catal. A: Chem. 2006 245,37 .

[23] Biswanath, D.; Katta, V.; Anjoy, M.; Majjigapu, R. J. Mol. Catal. A: Chem. 2006, 246, 276

[24] Hossein, E.; Seyed, M.; Elham, S.; Mohammad, V. J. Mol. Catal. A: Chem. 2012, 363, 430 .

[25] Mazaahir, K.; Saurav, B.; Neeraj, K.; Vikas, B. Catal. Commun. 2009, 10, 1514 .

[26] Kushal, D.; Pawan, J.; Kishor, P.; Bhalchandra, M. Catal. Commun. 2010, 11, 1233.

[27] Datta, B. Pasha, M. A. Phosphorus, Sulfur Silicon Relat. Elem. 2011, 186, 171.

[28] Andrade, C. K. Z.; Barreto, A. D. F. S.; Silva, W. A. ARKIVOC 2008, 226.

[29] Martins, M. A. P.; Rossatto, M.; Prola, L. D. T.; Pizzuti, L.; Moreira, D. N.; Campos, P. T.; Frizzo, C. P. Ultrason. Sonochem. 2012, 19, 227.

[30] Fan, W.-M.; Gao, J.-R.; Jia, J.-H. Chin. J. Org. Chem. 2010, 30, 1732 (in Chinese) (范文明，高建荣，贾建洪，有机化学， 2010, 30, 1732.)

[31] Jiang, S.-L.; Han, L. Chin. J. Org. Chem. 2012, 32, 930 (in Chinese). (蒋绍亮, 韩亮, 有机化学, 2012, 32, 930.)

[32] Velladurai, R.; Kuppusamy, K.; Kasi, P. Tetrahedron Lett. 2012, 53, 1018. 